\title{
A Predictive Processing Account of Bottom-Up Visual Saliency Using Cross-Predicting Autoencoders
}

\begin{abstract}
We propose a novel predictive processing account of bottom-up visual saliency in which salience is simply the low-level prediction error between the sense-data and the predictions produced by the generative models in the brain. We test this with modelling in which we use cross-predicting deep autoencoders to create salience maps in an entirely unsupervised way. The resulting maps closely mimic experimentally derived human saliency maps and also spontaneously learn a centre bias, a robust viewing behaviour seen in human participants.
\end{abstract}

Every second, humans receive a torrent of incoming sense data, only a tiny subset of which is consciously processed. The choice of what to look at in a scene defines visual saliency. Saliency is traditionally partitioned into two components: a fast, bottom-up component calculated directly from raw image statistics (Yarbus, 1967; Gao \& Vasconcelos, 2007), and a slower top-down component that modulates the bottom-up salience with information about specific task demands, motivational factors, and other high-level information (Torralba \& Oliva, 2003; Kanan et al., 2009). These two components interact. Highly salient features and stimuli can capture attention automatically (Bacon \& Egeth, 1994; Treisman \& Gelade, 1980), as does the abrupt onset or offset of a new and salient perceptual object (Egeth \& Yantis, 1997; Jonides \& Yantis, 1988), even when participants are explicitly instructed not to attend to it. On the other hand, top-down attention and expectation can influence the allocation of salience. Observers can volitionally select regions of space (Posner, 1980), and individual objects (Rock \& Gutman, 1981; Duncan, 1984) to attend to even in the absence of intrinsically salient stimuli. Moreover, as shown by Henderson (2017), under strong task demands such as visual search in a known environment - searching for a cup in a kichen scene - or for sports such as tennis, the top down modulation can completely dominate the 
bottom-up saliency derived from the image. For instance, when viewing a film of a tennis match, observers fixate the region where the ball is predicted to bounce on each serve, even though it is almost always grass, similar to all the other grass, and therefore lacking in any particular bottom-up salient features. As such, bottom-up saliency models should best be considered models of default, or free-viewing conditions, where the participants are exploring the scene without any particular purpose or task demands.

Early models of visual saliency typically followed the path laid down by Koch and Ullman (1987) and first implemented by Niebur and Koch (1996) and Itti et al. (1998) (Einhäuser et al., 2007; Itti \& Koch, 2001). Saliency is modelled as activity in each of a number of feature channels,individually representing an important statistical property of the input, such as colour, intensity, orientation, or motion cues. The channels are then averaged to create a salience map. Significant work has gone into developing this model, by adding channels such as a face channel (Cerf et al., 2008), and a text channel (Gao et al., 2014), or by learning the channel-combination function by least squares instead of a simple average (Zhao \& Koch, 2013). Other models include those based on spectral analysis (Hou et al., 2012; Schauerte \& Stiefelhagen, 2012; Hou \& Zhang, 2007), information theory (Klein \& Frintrop, 2011, 2012; Bruce \& Tsotsos, 2009) and Bayesian reasoning (Gao et al., 2009; Gao \& Vasconcelos, 2007).

Recently, new 'deep learning' methods have arisen based on Convolutional Neural Networks (CNNs). These models attempt an 'end-to-end' supervised prediction of the saliency map given the raw pixels of an image as input. Early attempts such as those by Hong et al. (2015) and Vig et al. (2014) simply used a pretrained CNN model (VGG-net) and then used an Support Vector Machine (SVM) trained on the features extracted in the final layer of the CNN to predict the saliency maps. This approach, after modification, can still yield stateof-the-art performance (Kümmerer et al., 2014). Other methods use specialised CNN or Long-Short-Term-Memory (LSTM) architectures such as the deep fully convolutional Deep- 
Fix (Kruthiventi et al., 2015), recurrent CNNs (Liu \& Han, 2016), or convolutional LSTMs (Cornia et al., 2016) to achieve state of the art performance. Meanwhile there is evidence that updated Itti and Koch-based models are still competitive (Frintrop et al., 2015). Unlike earlier models, deep learning architectures are not unsupervised, appear to discover opaque 'features' of the scene, and make little reference to biological or cognitive principles. Thus, although they closely approximate human performance on saliency benchmarks (Bylinskii et al., 2012), they do little to improve our understanding of the cognitive processes underlying visual saliency.

In contrast, we propose a novel, entirely unsupervised, and biologically inspired model based on the predictive processing $(\mathrm{PP})$ paradigm. In brief, we suggest that people automatically attend to those parts of the scene that are unpredictable, these necessarily being the most informative areas to inspect.

The predictive processing paradigm paradigm (Friston, 2010, 2009; Kilner et al., 2007) posits that instead of passively receiving and extracting statistical information from incoming sensory signals, the brain instead is constantly engaged in a process of trying to predict the immediate future state of the world (Clark, 2012, 2015). PP theorists propose that the brain is a hierarchy of probabilistic generative models. Each generative model receives input from the layer beneath it and priors from the layer above. Combining these, it makes predictions about its future inputs, and it adjusts these predictions to minimize its surprisal (or simply the entropy) about the sense-data it receives and the states it occupies.

$$
\begin{aligned}
H(s \mid m) & =-\int p(s \mid d, m) \log p(s \mid d, m) d s \\
& \propto \int_{0}^{T} \log p(s(t) \mid d(t), m) d t
\end{aligned}
$$

Since this quantity cannot be optimized directly, it is assumed that instead the variational 
upper bound, called the variational free-energy, between the sense-data and a mind-internal "recognition density" $q(s \mid d, \theta)$ parametrized by $\theta$, is instead minimized (Feldman \& Friston, 2010; Friston et al., 2006, 2010).

$$
F=-\log p(d \mid m)+\int q(s \mid d \theta) \log q(s \mid d, \theta) d q-\int q(s \mid d, \theta) \log p(s, d \mid m) d q
$$

Under Gaussian assumptions of the recognition density and prior, optimising this quantity by gradient descent leads to simple Hebbian and biologically plausible update rules (Feldman \& Friston, 2010).

We hypothesize that the main metric the brain uses to determine bottom-up salience is the prediction error of the lower visual areas. Feldman and Friston (2010) propose that attention is simply the precision (or 'gain') of the prediction error. This approach is entirely compatible with our own since with every new image, the objects of attention change, and thus the primary determinant of the precision is the gradient of the precision which, under the predictive processing theory, is directly proportional to the prediction error between the recognition density and the sense-data.

This approach is also sensible from an information-maximising bayesian approach. Intuitively, it makes sense that the regions of the image to look at, in order to gain the maximum information are those that are the least predictable. Additionally, those unpredictable regions are usually those with the high-level image statistics such as high-contrast (Reinagel \& Zador, 1999), high-variance (Krieger et al., 2000), and high local-symmetry (Privitera \& Stark, 2000) that are common in highly salient regions.

More generally, our approach is one of exploiting the predictive paradigm between brain regions, as opposed to its typical application by cognitive scientists to the relation between the subject and the object (Shillcock \& Bailes, 2018). Paradigms such as change blindness 
(Simons, 2000; Simons \& Rensink, 2005) have demonstrated that much of our visual consciousness depends on inference as opposed to direct, sustained contact with the world.

We corroborate this theory with modelling evidence. In the PP approach, predictions are the output of generative models in the brain. This model in the general form can be represented as:

$$
v=f(x, \theta)+z \sim N\left(z ; 0, \Sigma_{v}\right)
$$

We choose to parametrize this function by a deep generative neural network. We propose a model based upon the 'split-brain' deep convolutional autoencoder of Zhang et al. (2016) which learns an unsupervised representation of the input using cross-prediction, whereby the whole scene input is partitioned into channels and each of two parallel models is trained by attempting to predict the other channel's input. We tested the hypothesis that this model would produce prediction-error maps that closely resembled salience maps for a wide variety of natural scenes. Additionally, we tested a second hypothesis that the widely observed 'centre bias' in scene viewing would emerge naturally from the model's behaviour.

Across many studies and datasets, a pronounced centre bias in visual salience is observed. Viewers have a significant tendency to attend to the centre of an image on the screen rather than the outlying regions (Foulsham \& Underwood, 2008; Mannan et al., 1995; Parkhurst et al., 2002; Parkhurst \& Niebur, 2003). Some have argued that the bias could be caused by the standard experimental setup in which participants are placed centrally in front of the screen (Judd et al., 2009; Tatler \& Vincent, 2008) combined with the decreased sensitivity of the visual system in the periphery (Parkhurst et al., 2002), the motor bias in the sacaddic system that favours relatively small saccades (Bahill \& Stark, 1977), and the natural tendency of the eye to centre in its orbit (Paré \& Munoz, 2001; Tatler, 2007; Zambarbieri et al., 1995). However, oculomotor factors cannot entirely be the cause. Vitu et al. (2004) has shown that 
for reading the bias is towards the centre of the screen showing the image rather than the orbital centre of the straight head position. Instead it has been proposed that the bias arises from image feature distribution patterns. As human photographers typically contrive to put the main features of interest at the centre of the image (Parkhurst et al., 2002; Reinagel \& Zador, 1999), observers of these images will eventually learn a prior that the most salient regions tend to be in the centre, thus resulting in a centre bias. Combined with a motor preference towards small amplitude saccades, this situation makes the centre the ideal place for a first fixation to begin exploration of the image (Le Meur et al., 2006). Therefore, even in datasets specifically designed not to exhibit centre bias, it is likely that participants, coming to the task with a strong prior towards central fixations, will nevertheless exhibit siginificant centre bias.

Since centre bias likely arises as an organic result of participants learning a prior over image feature distributions, it should also arise naturally from a biologically plausible and realistic model of visual saliency. However, most current models of visual saliency instead treat centre bias as an exogenous prior, often a simple gaussian located at the centre, and grafted on post-hoc to the model's predictions. Many models derived from the original Itti and Koch model treat the centre bias in this way, as do some current state of the art deep learning methods such as DeepGaze (Kümmerer et al., 2014). Other models treat centre bias in a more sophisticated manner. Zhao and Koch (Zhao \& Koch, 2011, 2012) treat the centre bias as a dynamic process, shifting and reducing over time. However, they still model the centre bias as an exogenous process to be modelled separately rather than one emerging naturally out of the normal functioning of the model. Perhaps the most sophisticated treatment is in Kruthiventi et al. (2015)'s model, which learns a centre bias directly using spatially variable CNN filters. However, their model learns directly from example salience maps, making its centre biases still an engineered feature rather than an emergent property as in our model, in which the centre bias arises directly from unsupervised learning of just the visual input. 
Since we propose that the centre bias is a learned prior over observed image salience distributions, a critical aspect of our second hypothesis is that we predict that the centre bias should emerge naturally from the nature of the images and the normal functioning of the model, with no effort or exogenous factors being necessary to obtain such a bias.

\section{Method and Results}

We employed a cross-predicting deep convolutional autoencoder architecture, similar to that of Zhang et al. (2016). Deep convolutional networks are widely used in the machine learning community (Krizhevsky et al., 2012) and have led the field in the modelling of visual saliency over the last few years (Bylinskii et al., 2012).

Autoencoders are neural network models trained to recreate their input. They can thus form unsupervised representations learned directly from data instead of needing data paired with the 'correct' human data. Since recreating the data exactly (the identity mapping) is an easy task to learn, modellers typically introduce some transformation to the dataset such as noise (Vincent et al., 2008) or a dimensional-bottleneck (Hinton \& Salakhutdinov, 2006) to reduce the dimensionality of the space. We argue that autoencoders fit the requirements of predictive processing well, since they are generative models that learn unsupervised representations of the data with all the expressive power of deep neural networks. Furthermore, as well as temporal autoencoding, which is where the brain tries to predict its input at time $t+1$ given its input at time $t$, we predict that the brain may utilise the different channels of information available to it as a means of cross-prediction. The visual areas of the brain are replete with recurrent connectivity (Felleman \& Van, 1991) and this, as well as the merging of the input from the two eyes and corresponding visual hemifields affords ample neural scope for such cross-prediction to be implemented in neural circuitry. 
Here we model only the colour channels, as an example of visual cross-prediction. Each image is presented in the RGB colour space, and split into three colour channels: red, green, and blue. An example of an image split into its colour channels is presented below.
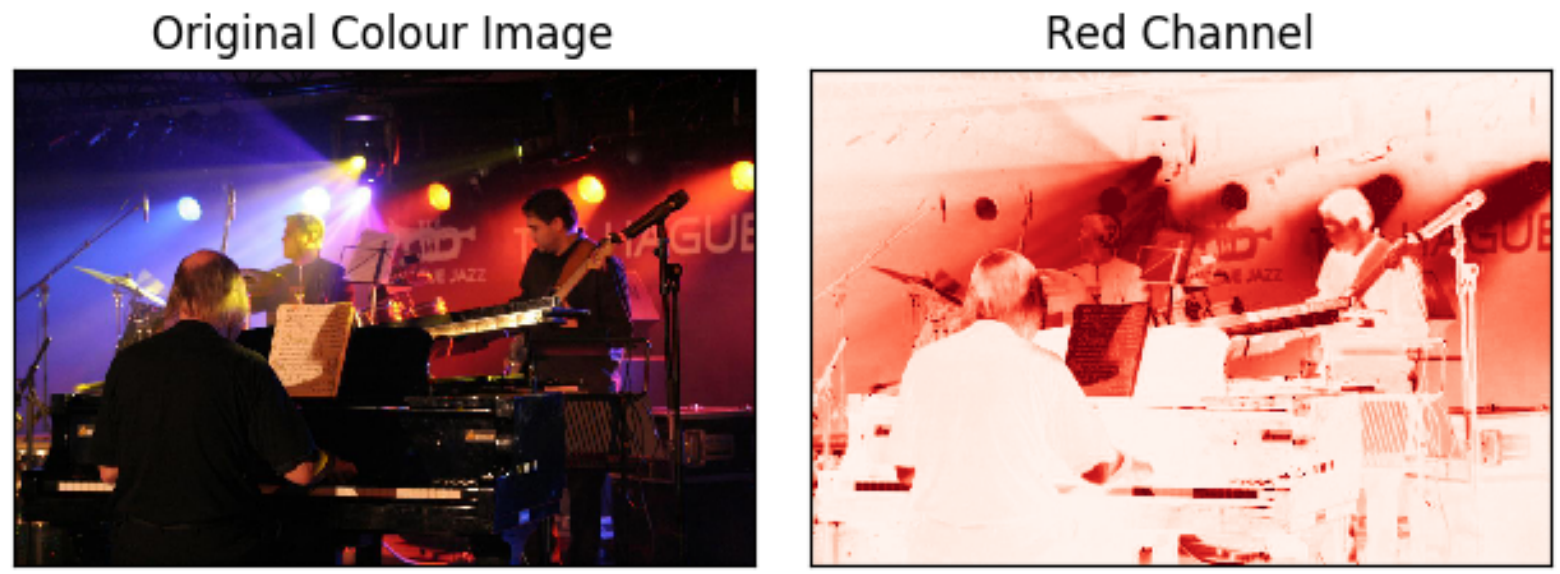

Green Channel
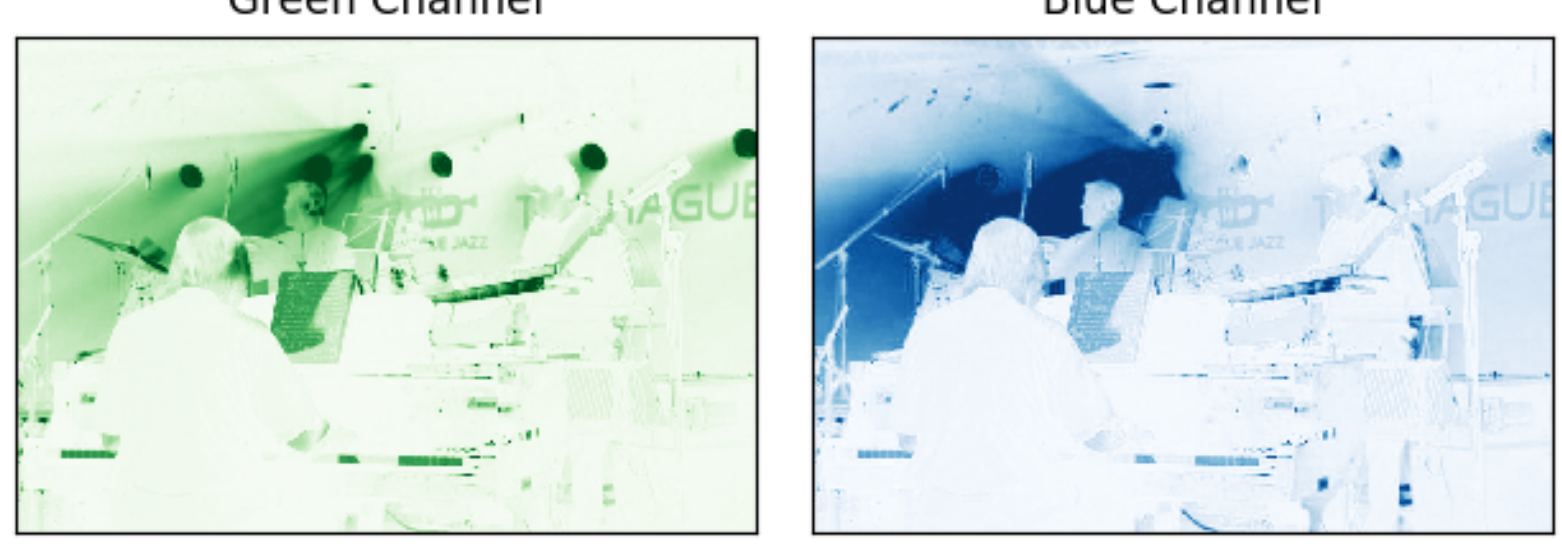

Figure 1: An example image with its red, green, and blue channels shown.

The cross-predicting autoencoder attempts to learn a mapping from one colourspace to the other. We hypothesise that learning these mappings forces the network to learn a representation of the latent structure of the image. This approach plausibly models visual cognition, since colour channels are processed separately and cross-prediction between these channels could enable image representations to be learned in an unsupervsied manner.

The network was composed in an encoder-decoder architecture with three convolutional 
blocks with batch-normalisation followed by a max-pooling layer. Dropout was applied with a dropout fraction of 0.1. Transposed convolutional layers were used in the decoder. The model was implemented in Keras. The convolutional layers had 32 or 64 feature maps, a typical kernel of $(2,2)$ and used either $(1,1)$ or $(2,2)$ stride widths. The max-pooling layer used a $2 \times 2$ pooling kernel. The model was trained using stochastic gradient descent with Nesterov momentum and a binary cross-entropy cost function. The initial learning rate was 0.001, and was halved upon the validation loss plateauing. For regularisation, a weight decay coefficient of 0.00001 was added.

The training set of images and saliency was sourced from the MIT benchmark dataset, comprising the MIT300 (Bylinskii et al., 2012) and the CAT2000 datasets (Borji \& Itti, 2015). The images were split into channels by colour. Each image was split into red green and blue channels (RGB). The network was trained to predict green images from blue, red from green, and vice versa.

The network was trained for 100 epochs on the training set, and then tested on the test set. For each test set image presented, the image was split into the two channels and then the image of one channel was presented to each of the subnetworks. The predictions were then compared with the other channel image. Error maps were created by subtracting the predicted image from the actual image channel, divided by the precision of the error, as in predictive processing. The precision of the error was calculated as the average variance of the prediction error across error maps. This step was taken since, in the predictive processing framework, under a stationary data ditribution, the precision converges to the variance of the prediction error. For each image, each network produced an error map. A master error map was created by a linear averaging of all the individual error maps.

$$
E_{i}=\frac{\left\|\hat{y}_{i}-y_{i}\right\|}{\Sigma_{i}}
$$




$$
\begin{gathered}
\Sigma_{i}=\left\langle\left(\hat{y}_{i}-y_{i}\right)^{2}\right\rangle \\
E=\frac{\sum_{i}^{N} E_{i}}{N}
\end{gathered}
$$

Examples of error maps, and the 'gold standard' saliency map for comparison are shown in Fig. 2.

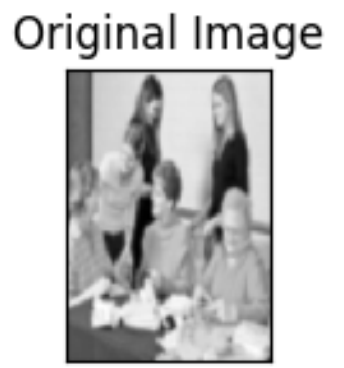

Original Image

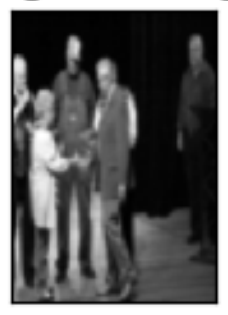

Original Image

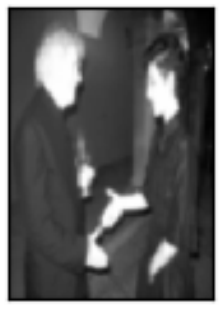

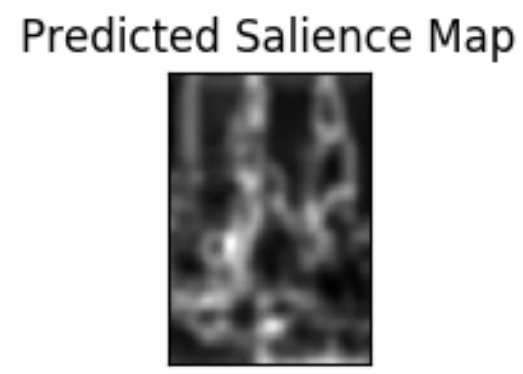

Predicted Salience Map

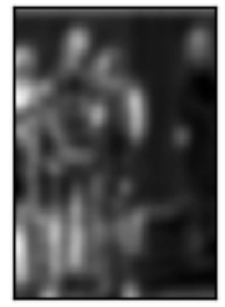

Predicted Salience Map

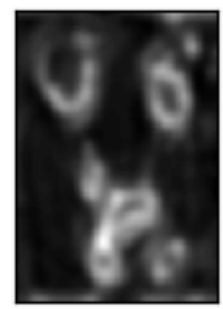

Actual Salience Map

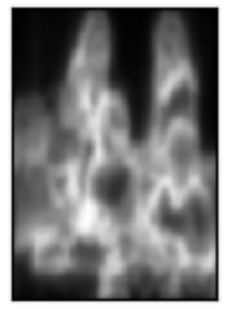

Actual Salience Map

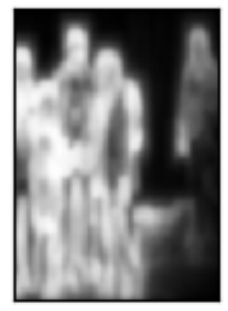

Actual Salience Map

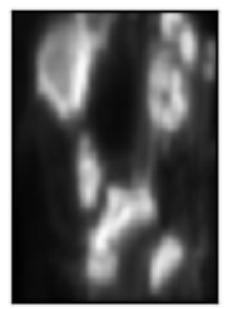

Figure 2: Example salience maps generated by the cross-predicting autoencoder.

The master error map, shown in Figure 3, allows us to test our second hypothesis, that the centre bias would emerge naturally from our model. It shows a clear centre bias. 


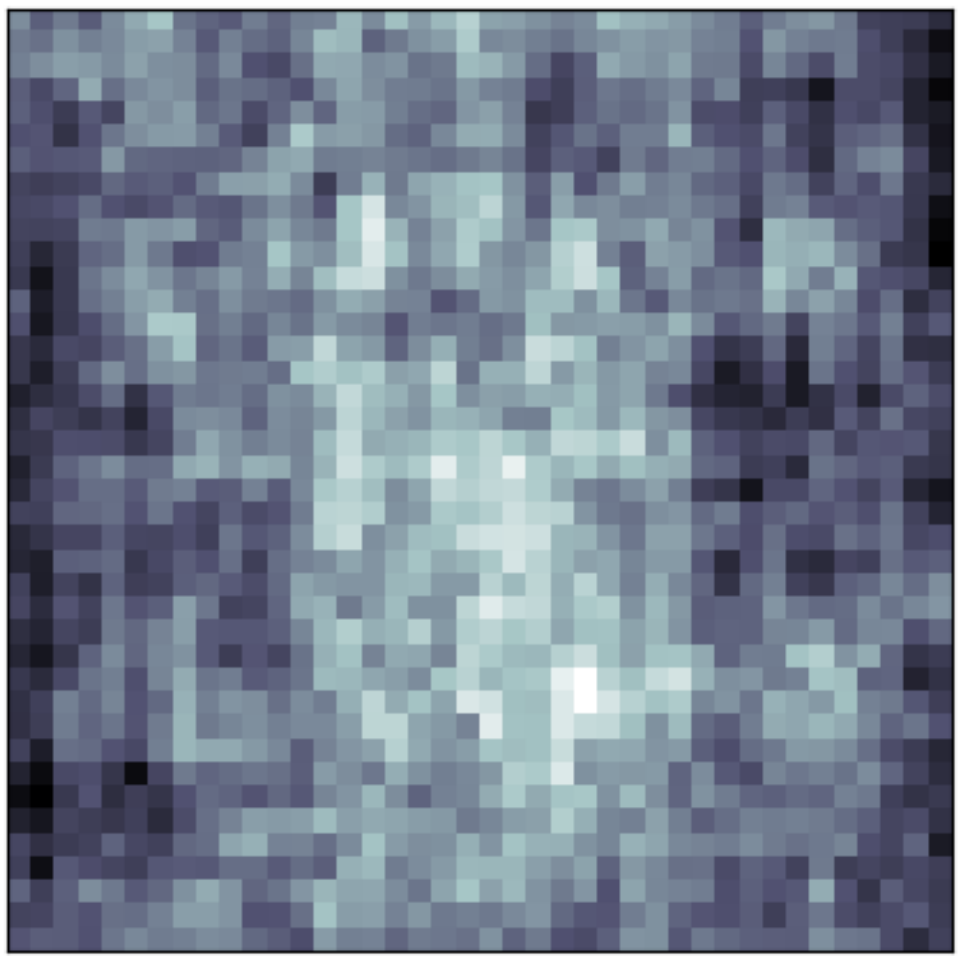

Figure 3: Master error map created by a linear averaging of all the individual error maps

\section{Discussion}

Our results show that cross-predicting autoencoders can generate realistic saliency maps in an entirely unsupervised way simply by attempting to predict different channels of the image. When averaged together these salience maps show a significant centre bias which has emerged naturally from the input statistics. Unlike both Itti-and-Koch-derived models, as well as the more recent deep learning techniques, our results are entirely unsupervised and, during training, no reference was made to the ground-truth human salience maps at all. Moreover, our results are firmly grounded in a well-supported cognitive paradigm, that of predictive processing, and additionally do not require the visual system to undertake any specific saliency processing, perhaps requiring dedicated neural circuitry. Instead, our system 
uses the prediction error as its bottom-up saliency measure, which, according to the theory of predictive processing, is calculated automatically at every level of the brain's hierarchy. Our model thus provides an elegant synthesis of predictive processing and bottom-up visual saliency, implemented in a cognitively plausible manner, and which generates salience maps and centre biases similar to the observed human norms.

In our study, the regions of the images with the largest prediction errors usually involved edges, regions of complex and variable terrain, or very detailed regions such as text or faces. This observation is consistent with other studies that have found that high-contrast, highvariance regions (Reinagel \& Zador, 1999) as well as those with distinctive higher-order statistics (Krieger et al., 2000) tend to attract human visual attention — precisely those regions that are picked out by the autoencoder as they are hard to predict. This behaviour can also be justified under an information-theoretic perspective as those regions that are difficult to predict will tend to be those that, when fixated upon and attended to, reveal the most information about the scene. Thus, focusing scarce attentional resources on the most unpredictable regions provides a reasonable approximating heuristic to the minimization of Bayesian surprise across the image.

One feature of our results, observable in Fig. 2, is that the error maps produced by our model typically have significantly more regions of salience than the human salience maps. One explanation for this fact is that the human 'saliency' maps employed as target data by saliency modellers are often operationalized as fixation maps, consisting of a number of sequential fixations. If our model was constrained to choose some fixed number of points to fixate, based on the most salient regions, then a sparser pattern would be observed.

Another interpretation of our model's behaviour, as seen in Fig. 2, is that it is effectively detecting edges. Edges have been argued to have a special status in object recognition (Bie- 
derman \& Ju, 1988); our modelling suggests a continuity between salience and edge-detection. Edge detection at a low-level is a natural consequence of predictive processing theory in vision since edges, being strongly discontinuous regions, are necessarily harder to predict than object continuations.

Although situated within a cognitively plausible paradigm, the specifics of the model itself are not particularly biologically plausible since it is a convolutional neural network trained by stochastic gradient descent. Convolutional networks, although inspired by neural processing in the visual system, are not biologically plausible in their specifics, and the question of whether the brain can learn through processes similar to stochastic gradient descent in artificial neural networks is controversial. Nevertheless, our model provides an existence proof of the utility of cross-predictive autoencoders for visual representation learning and supports our theory that good salience maps can be obtained simply from the prediction error of the brain's low-level generative models, and thus that visual saliency can thus be easily integrated into the overarching framework of predictive processing.

\section{Conclusion}

In this paper we have proposed a novel, parsimonious, predictive processing account of visual saliency - that it is simply the prediction error of the lower regions of the visual cortex. We have shown that this claim is in line with Friston (2010)'s argument that attention is simply the precision of the prediction error, and also that such a mechanism makes sense in an intuitive and information-theoretic sense. Furthermore, we have corroborated this theory with cognitively-plausible modelling, which has shown that a predictive model using deep convolutional cross-predicting autoencoders is able to generate realistic salience maps in an entirely unsupervised manner, and that these maps also naturally recreate, on average, the centre bias observed in human participants. 


\section{References}

Bacon, W. F. \& Egeth, H. E. (1994). Overriding stimulus-driven attentional capture. Perception 83 Psychophysics, 55(5):485-496.

Bahill, A. T. \& Stark, L. (1977). Oblique saccadic eye movements: Independence of horizontal and vertical channels. Archives of Ophthalmology, 95(7):1258-1261.

Biederman, I. \& Ju, G. (1988). Surface versus edge-based determinants of visual recognition. Cognitive Psychology, 20(1):38-64.

Borji, A. \& Itti, L. (2015). Cat2000: A large scale fixation dataset for boosting saliency research. CVPR 2015 Workshop on "Future of Datasets". arXiv preprint arXiv:1505.03581.

Bruce, N. D. \& Tsotsos, J. K. (2009). Saliency, attention, and visual search: An information theoretic approach. Journal of Vision, 9(3):5-5.

Bylinskii, Z., Judd, T., Borji, A., Itti, L., Durand, F., Oliva, A., \& Torralba, A. (2012). Mit saliency benchmark.

Cerf, M., Harel, J., Einhäuser, W., \& Koch, C. (2008). Predicting human gaze using lowlevel saliency combined with face detection. In Advances in Neural Information Processing Systems, pages 241-248.

Clark, A. (2012). Dreaming the whole cat: Generative models, predictive processing, and the enactivist conception of perceptual experience. Mind, 121(483):753-771.

Clark, A. (2015). Surfing uncertainty: Prediction, action, and the embodied mind. Oxford University Press.

Cornia, M., Baraldi, L., Serra, G., \& Cucchiara, R. (2016). Predicting human eye fixations via an lstm-based saliency attentive model. arXiv preprint arXiv:1611.09571. 
Duncan, J. (1984). Selective attention and the organization of visual information. Journal of Experimental Psychology: General, 113(4):501.

Egeth, H. E. \& Yantis, S. (1997). Visual attention: Control, representation, and time course. Annual Review of Psychology, 48(1):269-297.

Einhäuser, W., Mundhenk, T. N., Baldi, P., Koch, C., \& Itti, L. (2007). A bottom-up model of spatial attention predicts human error patterns in rapid scene recognition. Journal of Vision, 7(10):6-6.

Feldman, H. \& Friston, K. J. (2010). Attention, uncertainty, and free-energy. Frontiers in Human Neuroscience, 4.

Felleman, D. J. \& Van, D. E. (1991). Distributed hierarchical processing in the primate cerebral cortex. Cerebral Cortex (New York, NY: 1991), 1(1):1-47.

Foulsham, T. \& Underwood, G. (2008). What can saliency models predict about eye movements? spatial and sequential aspects of fixations during encoding and recognition. Journal of Vision, 8(2):6-6.

Frintrop, S., Werner, T., \& Martin Garcia, G. (2015). Traditional saliency reloaded: A good old model in new shape. In Proceedings of the IEEE Conference on Computer Vision and Pattern Recognition, pages 82-90.

Friston, K. (2009). The free-energy principle: a rough guide to the brain? Trends in Cognitive Sciences, 13(7):293-301.

Friston, K. (2010). The free-energy principle: a unified brain theory? Nature Reviews Neuroscience, 11(2):127-138.

Friston, K., Kilner, J., \& Harrison, L. (2006). A free energy principle for the brain. Journal of Physiology-Paris, 100(1):70-87. 
Friston, K. J., Daunizeau, J., Kilner, J., \& Kiebel, S. J. (2010). Action and behavior: a free-energy formulation. Biological Cybernetics, 102(3):227-260.

Gao, D., Han, S., \& Vasconcelos, N. (2009). Discriminant saliency, the detection of suspicious coincidences, and applications to visual recognition. IEEE Transactions on Pattern Analysis and Machine Intelligence, 31(6):989-1005.

Gao, D. \& Vasconcelos, N. (2007). Bottom-up saliency is a discriminant process. In Computer Vision, 200\%. ICCV 200\%. IEEE 11th International Conference on, pages 1-6. IEEE.

Gao, R., Uchida, S., Shahab, A., Shafait, F., \& Frinken, V. (2014). Visual saliency models for text detection in real world. PLoS one, 9(12):e114539.

Henderson, J. M. (2017). Gaze control as prediction. Trends in Cognitive Sciences, 21(1):1523.

Hinton, G. E. \& Salakhutdinov, R. R. (2006). Reducing the dimensionality of data with neural networks. Science, 313(5786):504-507.

Hong, S., You, T., Kwak, S., \& Han, B. (2015). Online tracking by learning discriminative saliency map with convolutional neural network. In International Conference on Machine Learning, pages 597-606.

Hou, X., Harel, J., \& Koch, C. (2012). Image signature: Highlighting sparse salient regions. IEEE Transactions on Pattern Analysis and Machine Intelligence, 34(1):194-201.

Hou, X. \& Zhang, L. (2007). Saliency detection: A spectral residual approach. In Computer Vision and Pattern Recognition, 200\%. CVPR'0\%. IEEE Conference on, pages 1-8. IEEE.

Itti, L. \& Koch, C. (2001). Computational modelling of visual attention. Nature Reviews Neuroscience, 2(3):194. 
Itti, L., Koch, C., \& Niebur, E. (1998). A model of saliency-based visual attention for rapid scene analysis. IEEE Transactions on Pattern Analysis and Machine Intelligence, 20(11):1254-1259.

Jonides, J. \& Yantis, S. (1988). Uniqueness of abrupt visual onset in capturing attention. Perception \& Psychophysics, 43(4):346-354.

Judd, T., Ehinger, K., Durand, F., \& Torralba, A. (2009). Learning to predict where humans look. In Computer Vision, 2009 IEEE 12th International Conference on, pages 2106-2113. IEEE.

Kanan, C., Tong, M. H., Zhang, L., \& Cottrell, G. W. (2009). Sun: Top-down saliency using natural statistics. Visual Cognition, 17(6-7):979-1003.

Kilner, J. M., Friston, K. J., \& Frith, C. D. (2007). Predictive coding: an account of the mirror neuron system. Cognitive Processing, 8(3):159-166.

Klein, D. A. \& Frintrop, S. (2011). Center-surround divergence of feature statistics for salient object detection. In Computer Vision (ICCV), 2011 IEEE International Conference on, pages 2214-2219. IEEE.

Klein, D. A. \& Frintrop, S. (2012). Salient pattern detection using w2 on multivariate normal distributions. In Joint DAGM (German Association for Pattern Recognition) and OAGM Symposium, pages 246-255. Springer.

Koch, C. \& Ullman, S. (1987). Shifts in selective visual attention: towards the underlying neural circuitry. In Matters of Intelligence, pages 115-141. Springer.

Krieger, G., Rentschler, I., Hauske, G., Schill, K., \& Zetzsche, C. (2000). Object and scene analysis by saccadic eye-movements: an investigation with higher-order statistics. Spatial Vision, 13(2):201-214. 
Krizhevsky, A., Sutskever, I., \& Hinton, G. E. (2012). Imagenet classification with deep convolutional neural networks. In Advances in Neural Information Processing Systems, pages 1097-1105.

Kruthiventi, S. S., Ayush, K., \& Babu, R. V. (2015). Deepfix: A fully convolutional neural network for predicting human eye fixations.corr abs/1510.02927 (2015).

Kümmerer, M., Theis, L., \& Bethge, M. (2014). Deep gaze i: Boosting saliency prediction with feature maps trained on imagenet. arXiv preprint arXiv:1411.1045.

Le Meur, O., Le Callet, P., Barba, D., \& Thoreau, D. (2006). A coherent computational approach to model bottom-up visual attention. IEEE Transactions on Pattern Analysis and Machine Intelligence, 28(5):802-817.

Liu, N. \& Han, J. (2016). A deep spatial contextual long-term recurrent convolutional network for saliency detection. arXiv preprint arXiv:1610.01708.

Mannan, S., Ruddock, K., \& Wooding, D. (1995). Automatic control of saccadic eye movements made in visual inspection of briefly presented 2-d images. Spatial Vision, 9(3):363386.

Niebur, E. \& Koch, C. (1996). Control of selective visual attention: Modeling the" where" pathway. In Advances in Neural Information Processing Systems, pages 802-808.

Paré, M. \& Munoz, D. P. (2001). Expression of a re-centering bias in saccade regulation by superior colliculus neurons. Experimental Brain Research, 137(3):354-368.

Parkhurst, D., Law, K., \& Niebur, E. (2002). Modeling the role of salience in the allocation of overt visual attention. Vision Research, 42(1):107-123.

Parkhurst, D. J. \& Niebur, E. (2003). Scene content selected by active vision. Spatial Vision, 16(2):125-154. 
Posner, M. I. (1980). Orienting of attention. Quarterly Journal of Experimental Psychology, $32(1): 3-25$.

Privitera, C. M. \& Stark, L. W. (2000). Algorithms for defining visual regions-of-interest: Comparison with eye fixations. IEEE Transactions on Pattern Analysis and Machine Intelligence, 22(9):970-982.

Reinagel, P. \& Zador, A. M. (1999). Natural scene statistics at the centre of gaze. Network: Computation in Neural Systems, 10(4):341-350.

Rock, I. \& Gutman, D. (1981). The effect of inattention on form perception. Journal of Experimental Psychology: Human Perception and Performance, 7(2):275.

Schauerte, B. \& Stiefelhagen, R. (2012). Quaternion-based spectral saliency detection for eye fixation prediction. Computer Vision-ECCV 2012, pages 116-129.

Shillcock, R. \& Bailes, R. (2018). Mirror neurons, prediction and hemispheric coordination; the prioritizing of intersubjectivity over 'intrasubjectivity'. Under Review.

Simons, D. J. (2000). Current approaches to change blindness. Visual Cognition, 7(1-3):1-15.

Simons, D. J. \& Rensink, R. A. (2005). Change blindness: Past, present, and future. Trends in Cognitive Sciences, 9(1):16-20.

Tatler, B. W. (2007). The central fixation bias in scene viewing: Selecting an optimal viewing position independently of motor biases and image feature distributions. Journal of Vision, $7(14): 4-4$.

Tatler, B. W. \& Vincent, B. T. (2008). Systematic tendencies in scene viewing. Journal of Eye Movement Research, 2(2).

Torralba, A. \& Oliva, A. (2003). Statistics of natural image categories. Network: Computation in Neural Systems, 14(3):391-412. 
Treisman, A. M. \& Gelade, G. (1980). A feature-integration theory of attention. Cognitive Psychology, 12(1):97-136.

Vig, E., Dorr, M., \& Cox, D. (2014). Large-scale optimization of hierarchical features for saliency prediction in natural images. In Proceedings of the IEEE Conference on Computer Vision and Pattern Recognition, pages 2798-2805.

Vincent, P., Larochelle, H., Bengio, Y., \& Manzagol, P.-A. (2008). Extracting and composing robust features with denoising autoencoders. In Proceedings of the 25th International Conference on Machine Learning, pages 1096-1103. ACM.

Vitu, F., Kapoula, Z., Lancelin, D., \& Lavigne, F. (2004). Eye movements in reading isolated words: evidence for strong biases towards the center of the screen. Vision Research, $44(3): 321-338$.

Yarbus, A. (1967). Eye movement and vision.

Zambarbieri, D., Beltrami, G., \& Versino, M. (1995). Saccade latency toward auditory targets depends on the relative position of the sound source with respect to the eyes. Vision Research, 35(23):3305-3312.

Zhang, R., Isola, P., \& Efros, A. A. (2016). Split-brain autoencoders: Unsupervised learning by cross-channel prediction. arXiv preprint arXiv:1611.09842.

Zhao, Q. \& Koch, C. (2011). Learning a saliency map using fixated locations in natural scenes. Journal of Vision, 11(3):9-9.

Zhao, Q. \& Koch, C. (2012). Learning visual saliency by combining feature maps in a nonlinear manner using adaboost. Journal of Vision, 12(6):22-22.

Zhao, Q. \& Koch, C. (2013). Learning saliency-based visual attention: A review. Signal Processing, 93(6):1401-1407. 